\title{
Recognition of Indian Paper Currency based on LBP
}

\author{
Bhawani Sharma \\ Assistant Professor \\ Department of Computer \\ Science, Harf College, MLK, \\ Sangrur, Punjab, India
}

\author{
Amandeep Kaur \\ Associate Professor \\ Department of Computer \\ Science, Punjabi University, \\ Patiala, Punjab, India
}

\author{
Vipan \\ Assistant Professor \\ Department of Computer \\ Science, R.K. Arya College, \\ Nawanshahr, Punjab,India
}

\begin{abstract}
The importance of automatic methods has increased greatly for paper currency recognition due to the development of modern banking services like automated teller machine (ATM) and automatic goods seller machines. For a machine, it is a challenge to recognize paper currency whether it is of $10,20,50,100,500$ or 1000 . Various method or algorithm has been applied to overcome this challenge and in most of systems neural network is used. For this, LBP algorithm is proposed in this paper. The proposed method has advantages of simplicity and high speed. The experimental results show that this method has a high recognition rate. The recognition ratio can achieve $100 \%$ in case of good quality images.
\end{abstract}

\section{General Terms}

Digital Image Processing

\section{Keywords}

Feature extraction, LBP algorithm, paper currency recognition.

\section{INTRODUCTION}

Due to development of automated systems the importance for automatic methods for currency recognition has increased day by day. An efficient currency recognition system is vital for the automation in many sectors such as vending machine, railway ticket counter, banking system, shopping mall, currency exchange service etc. Automatic machines capable of recognizing banknotes are massively used in automatic dispensers of a number of different products, ranging from cigarettes to bus tickets, as well as in many automatic banking operations. For instance, most machines operating in automatic dispensers are not very effective in terms of performance, but they are available at very low cost, whereas machines used in banking operations usually have to pass very severe tests, but are available at a higher cost. The needs for an automatic banknote recognition system encouraged many researchers to develop robust and reliable techniques [18]. Speed and accuracy of processing are two important factors in such systems.

Paper currency recognition systems should be able to recognize banknotes from each side and each direction. Since banknotes may be defected during circulation, the designed system should have a meaningful accuracy in detecting torn or worn banknotes.

Until now, there are many methods proposed for paper currency recognition. The simplest way is to make use of the visible features of the paper currency. Like the size and texture of the paper currency [9]. However, this kind of methods has great limitations as currencies of different values may have the same size in some countries, and the visible marks may be contaminated by noise. In paper currency recognition, Neural Network is widely used. Takeda F. et al [2] have used two types of data set $\&$ a random mask for preprocessing the data and used a multi-layer neural network as the classifier for recognition of paper currency. Pathrabe T. et al [10] used HSV technique for extraction of the features and classifier based on Neural Network.

Although the NN [1-8] technology has the ability of selforganization, generalization and parallel processing, and has a good fit for pattern recognition, it also has some weakness. First, it needs a large number of training samples, which are used to avoid over-fitting and poor generalization. Second, if the distribution of training sample is not uniform, the result will probably converge to a local optimal or will even diverge unreasonably. The examples must be selected carefully otherwise useful time is wasted or even worse the network might be functioning incorrectly. The disadvantage is that because the network finds out how to solve the problem by itself, its operation can be unpredictable. Therefore, the selection of the training set is a crucial issue for the NN. In case of paper currency, complex designs of different kinds of paper currencies make automatic currency recognition difficult to work well. Sometimes the original information on paper currency may have a loss because paper currency may be worn, blurry, or even damaged. So it is important how to extract the characteristic information from currency image and select proper pattern recognition algorithms to improve the accuracy of currency recognition.

In this thesis work, LBP (Local Binary Pattern) is used for feature extraction purpose. Initially LBP was introduced as texture analysis technique $[11,12]$. It is highly used for texture analysis and feature extraction. In paper currency, many types of texture are used. We can treat different types of currencies as images with different textures, thus we can extract the texture features for recognition. LBP is a powerful tool for texture description. The algorithm has low computational complexity, which can meet the high speed requirement in practical applications.

It needs to be noted that the proposed technique may not be able to distinguish counterfeit notes from genuine notes. Indeed, techniques such as $[13,14]$ which use infrared or ultraviolet spectra may be used for discriminating between genuine and counterfeits notes.

Remaining part of this paper is as follows. In section 2 and 3, we briefly introduce the Local Binary Pattern and LBP image. Section 4 presents the proposed algorithm and Section 5 includes experiments and results. Finally, conclusions are drawn in section 6 . 


\section{Local Binary Pattern}

In this section, we introduce the original LBP operator.

The basic LBP operator is first introduced by Ojala T. et al. [15]. LBP is a powerful tool for texture description. Now these days, LBP has been applied in various fields like Fingerprint Recognition, Face Recognition, Facial Expression, 3D face recognition, Medical Imaging. In LBP, the neighborhood pixels are converted to binary code 0 or 1 by using the gray value of the center pixel as threshold, and then all these codes form an ordered pattern according to their positions relative to the center pixel.

Let $g_{c}$ be the gray value of the center pixel $p$, and $g_{i}$ be the gray value of the $i^{\text {th }}$ pixel in clockwise order at the 8 neighborhood of pixel $\mathrm{i}$, where $i=0,1, \ldots 7$. Now the LBP code for pixel $\mathrm{p}$ is defined as equation (1)

$$
\operatorname{LBP}_{(p)}=\sum_{i=0}^{7} 2^{i} \times S\left(g_{i}-g_{c}\right)
$$

Where $\mathrm{S}(\mathrm{x})$ is the threshold function that can be defined as equation (2)

$$
S(x)= \begin{cases}1 & \text { for } x \geq 0 \\ 0 & \text { otherwise }\end{cases}
$$

Following fig (1) is detailed illustration about this process.

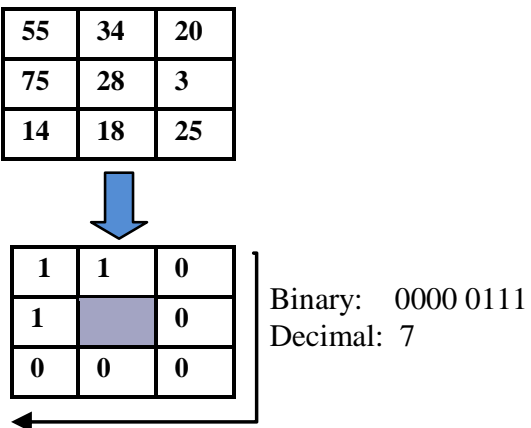

Fig 1: Illustration of LBP operator

We can see from Equation (1) that LBP operator calculates the relative difference value, so that it has gray-value invariance. LBP can produce 256 kinds of different outputs, corresponding to 256 kinds of different binary patterns. The best advantage of LBP is its simple computation, which can be applied in the situation where high speed is needed. The feature extracted with LBP reflects the relationship of the texture within local area.

\section{LBP image}

Given a grayscale image $I$, scan every pixel in a raster order: the LBP code for each pixel $p$ of image $I$ can be calculated according to equation (1), therefore the resulted LBP image $I_{L B P}$ is obtained.
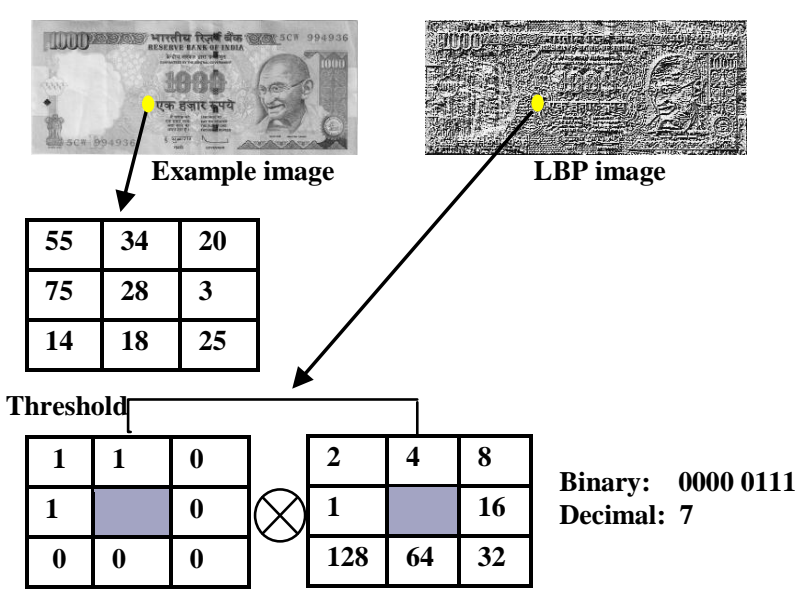

Fig 2: Example of how the LBP operator works.

See Fig 2 for an illustration of how the original LBP operator works and how an LBP image is produced.

Histogram is a widely used feature to describe the global characteristic of an image. LBP histogram of an image is a 256-dimensional vector. The value of each dimension $i$ ( $i=0$, $1,2,3 \ldots .255)$ indicates the number of pixels which have LBP value of $i$.

\section{Proposed Algorithm}

In this section we describe the implementation of paper currency recognition system using our proposed method. Following are the main steps.

1. Prepare the paper currency images by acquisition. For example, we consider 6 kinds of paper currencies for recognition, including $10,20,50,100,500$ and 1000 rupees. For each bank denomination there are 4 directions for inserting it into a machine, such as A, B, C, $\mathrm{D}$ as shown in Figure 3. So the total number of categories considered by the classifier is 24 .

2. Feature extraction. The feature vectors (i.e. the models) of all images are saved and will be used during classification purposes.

3. Read the test sample image of the paper currency and extract its feature as described above.

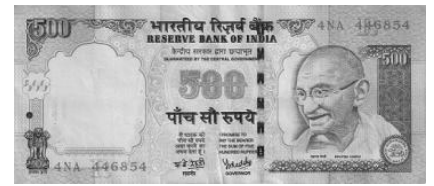

(A) Front side - Normal direction

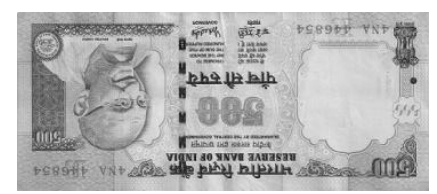

(B) Front side - reverse direction 


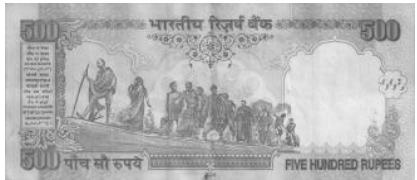

(C) Back side - Normal direction

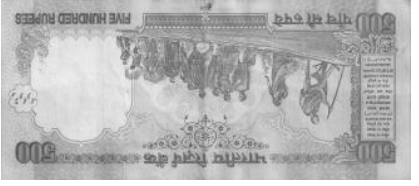

(D) Back side - reverse direction

Fig 3: Four directions of inserting a paper currency

4. Calculate the similarity between the sample image and each template image according to equation (3). The sample is classified to the class whose template is the most similar one with the sample.

For recognition of Indian paper currency algorithm, we adopt Euclidean distance as classifier.

E.D. $=\sqrt{\sum_{i=0}^{n}\left(\mathrm{x}_{\mathrm{i}}-\mathrm{y}_{\mathrm{i}}\right)^{2}}$

\section{Experiments and Results}

In the experiments, we tested approximately 504 sheets of Indian paper currency (132 sheets of 10rupees, 32 sheets of 20rupees, 68 sheets of 50rupees, 116 sheets of 100rupees, 112 sheets of 500rupees, 44 sheets of 1000rupees). For each side and each direction, there are new and old traditional paper currencies involved. These test sample images were obtained from the paper currencies by a scanner. The recognition result is listed in the Table (1). The recognition ratio can achieve $100 \%$ in case of good quality images.

The performance of proposed algorithm is evaluated on Indian paper currency image database. During training phase, features of all training set images are averaged in a particular expression class to generate a trained feature vector for that particular class. In testing phase the Euclidean Distance is used to find the closest histogram feature template to the query image. The result shows that recognition rate of the proposed algorithm is comparable or better than the existing techniques.

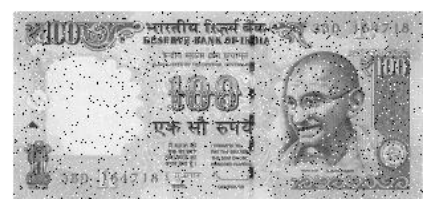

Fig 4: Add the Salt and Pepper noise with density of 0.02 .

In order to test our algorithm on paper currency which is contaminated during the circulation or image acquisition process, we apply salt and pepper noise to the original samples as shown in above Fig (4) to simulate poor quality paper currency. Matlab 10.0 is used to accomplish this task. In case of salt and pepper noise, varying illumination, it is still able to obtain recognition rate of $98 \%$. In case of low noise level, this method can be applied but as the noise is increased in paper currency images, recognition rate decreased which cannot be neglected. This method doesn't provide good results, if paper currency is blurred i.e Gaussion noise is present.

Table 1: Recognition rate of good quality images.

\begin{tabular}{|c|c|c|c|c|c|c|c|}
\hline \multicolumn{2}{|c|}{ Currency Type } & $\mathbf{1 0}$ & $\mathbf{2 0}$ & $\mathbf{5 0}$ & $\mathbf{1 0 0}$ & $\mathbf{5 0 0}$ & $\mathbf{1 0 0 0}$ \\
\hline & $\begin{array}{c}\text { Currency } \\
\text { (Normal) }\end{array}$ & 100 & 100 & 100 & 100 & 100 & 100 \\
\cline { 2 - 7 } $\begin{array}{c}\text { Recognition } \\
\%\end{array}$ & $\begin{array}{c}\text { Currency } \\
\text { ( Old } \\
\text { tradition) }\end{array}$ & 100 & 100 & 100 & 100 & 100 & 100 \\
\hline
\end{tabular}

Table 2: Recognition rate of same denominations.

\begin{tabular}{|c|c|c|c|c|c|c|}
\hline Bank Note & $\mathbf{1 0}$ & $\mathbf{2 0}$ & $\mathbf{5 0}$ & $\mathbf{1 0 0}$ & $\mathbf{5 0 0}$ & $\mathbf{1 0 0 0}$ \\
\hline $\begin{array}{c}\text { Recognitio } \\
\mathrm{n} \%\end{array}$ & 100 & 99 & 100 & 100 & 100 & 100 \\
\hline $\begin{array}{c}\text { LBP Value } \\
\text { (min.) }\end{array}$ & 507.73 & $\begin{array}{c}498.0 \\
9\end{array}$ & $\begin{array}{c}541.1 \\
1\end{array}$ & $\begin{array}{c}510.3 \\
3\end{array}$ & $\begin{array}{c}453 . \\
89\end{array}$ & $\begin{array}{c}534 . \\
42\end{array}$ \\
\hline $\begin{array}{c}\text { LBP Value } \\
\text { (max.) }\end{array}$ & 777.69 & $\begin{array}{c}729.8 \\
5\end{array}$ & $\begin{array}{c}751.8 \\
8\end{array}$ & $\begin{array}{c}685.0 \\
8\end{array}$ & $\begin{array}{c}661 . \\
3\end{array}$ & $\begin{array}{c}643 . \\
32\end{array}$ \\
\hline
\end{tabular}

Above Table (2) shows its experimental results including LBP value When the paper currency with its same denomination is taken (i.e. banknote of Rs. 10 is compared with only set of banknotes of Rs. 10).

From the experimental results, we can see that on training process, we don't need large amount of samples for learning, which can save the training time.

\section{Conclusions}

In this paper, we have proposed an algorithm based on LBP for recognition of Indian paper currency. The experimental results have shown effectiveness of the proposed method. It is worth mentioning that our method has good performance for low noise, which is significant in practice. As the LBP operator has the advantage of simplicity, low computation complexity, which makes it possible to analyze images in challenging real-time settings and can satisfy the requirement of practical applications. We tested the effectiveness of our method on Indian paper currency. By preparing database of other countries, this method can be further used to recognize the others types of paper currency.

\section{ACKNOWLEDGMENTS}

The authors would like thanks Dr. Chandan Singh, Professor in Department of Computer Science, Punjabi University, Patiala for their valuable support and they also owe a special word of thanks to the Head of the Department of Computer Science and Engineering, Punjabi University, Patiala for providing infrastructure required in the proposed research work. 


\section{References}

[1] Takeda F. and Omatu S. , Onami S. , Kadono T. and Terada K,"A Paper Currency Recognition Method by a Small Size Neural Network with Optimized Masks by GA", Proceedings of IEEE World Congress on Computational Intelligence, 1994, 42434-246.

[2] Takeda F. and Omatu S, "High-speed paper currency recognition by neural networks," IEEE Trans Neural Networks, Vol: 6, Jan, 1995, 73-77.

[3] Takeda F. and Omatu S., "A Neuro-Money Recognition Using Optimized Masks by GA", Proceedings of IEEE International Conference on Systems, Vo1: 5, 1995, 4367-4371.

[4] Frosini A. ; Gori M. and Priami P. "A Neural NetworkBased Model For Paper Currency Recognition And Verification", IEEE Transactions On Neural Network, Vol: 7, Nov1996 Issue: 6, 1482-1490.

[5] Tanaka M. ; Takeda F. ; Ohkouchi K. and Michiyuki Y. "Recognition Of Paper Currencies By Hybrid Neural Network", IEEE Transactions On Neural Network, Vol: 3, 1998, 1748-1753.

[6] Takeda F. and Nishikage T. ," Multiple Kinds Of Paper Currency Recognition Using Neural Network And Application For Euro Currency", IEEE Transactions On Neural Network, Vol.2, 2000, 143-147.

[7] Zhang H, Jiang B.,Duan J.H and Bian Z.Z, “ Research on Paper Currency Reconition by Neural Networks", Proceedings of the 2nd Int. Conference on Machine Learning and Cybernetics, 2003.

[8] Kang J. and Kim H. "New Recognition Algorithm for Various Kinds of Euro Banknotes" Vol: 3, 2003, 22662270 .
[9] Hassanpour, H. ; Yaseri, A. and Ardeshiri, G. , "Feature Extraction For Paper Currency Recognition", IEEE Transactions On Signal Processing And Its Applications, 2007, 1-4.

[10] Pathrabe T. , Karmore S. ,"A Novel Approach of Embedded System for Indian Paper Currency Recognition", IJCTT , 2011

[11] Ojala T., Pietikinen M., and Harwood D., "A comparative study of texture measures with classification based on featured distribution", Pattern Recognition, Vol: 29, issue: 1, 1996.

[12] Ojala T., Pietikinen M., and Menp T., "Multiresolution grayscale and rotation invariant texture classification with local binary patterns", IEEE PAMI, Vol: 24, issue: 7, July 2002.

[13] A. Vila A., Ferrer N., Mantec'on J. , Bret'on D., Garc'ia J.F., "Development of a fast and nondestructive procedure for characterizing and distinguishing original and fake euro notes", Analytica Chimica Act., Vol: 559,2006, 257-263.

[14] Liu C, Ruan S and Huang G., "Research on identification the counterfeit by recognizing the infrared images", Vol: 4, 2008, 2081-2084.

[15] Ojala T., Pietikäinen M., and Harwood D., "A comparative study of texture measures with classification based on feature distributions," Pattern Recognition, Vol: 29, issue: 1, 1996, 51-59.

[16] R. C. Gonzalez and R. E. Woods, Digital image processing (Prentice Hall India, 2nd ed., 2006).

[17] A.K.Jain. Fundamentals of digital image processing. (Englewood Cliffs, NJ: Prentice-Hall, 1989). 